Proyecciones Journal of Mathematics

Vol. 29, No 3, pp. 291-307, December 2010.

Universidad Católica del Norte

Antofagasta - Chile

\title{
FINITE TOPOLOGIES AND DIGRAPHS
}

\author{
CARLOS MARIJUÁN \\ UNIVERSIDAD DE VALLADOLID, ESPANAA \\ Received: October 2010. Accepted : November 2010
}

\begin{abstract}
In this paper we study the relation between finite topologies and digraphs. We associate a digraph to a topology by means of the "specialization" relation between points in the topology. Reciprocally, we associate a topology to each digraph, taking the sets of vertices adjacent (in the digraph) to $v$, for all vertex $v$, as a subbasis of closed sets for the topology.

We use these associations to examine the relation between a simple digraph and its homologous topology. We also extend this relation to the functions preserving the structure between these classes of objects.
\end{abstract}

AMS Classification : 54A05, 05C20.

Keywords : Finite topologies, digraphs. 


\section{Introduction}

The one to one correspondence between finite preorder relations and finite topologies with the same underlying set of points, and also between finite posets and finite $T_{0}$ topologies is well known. Then, the one to one correspondence between finite digraphs and topologies is easily deducible. In fact, Evans, Harary and Lynn [3] prove that "There is a 1-1 correspondence between the labeled topologies with $n$ points and the labeled transitive digraphs with $n$ points". They associate a transitive digraph $D(T)$ to a topology $T$ with the same set of points as follows: "For two distinct points $u$ and $v$ of $T, u$ will be adjacent to $v$ in $D(T)$ provided $u$ is in every neighborhood of $v$ ". Reciprocally, "to each labeled transitive digraph $D$ with $n$ points, there corresponds a unique labeled topology $T(D)$, in which the basic open sets are the sets of points adjacent to $v$, for all points $v$ ".

In this paper, we study the relations between finite topologies and digraphs in a different way. We associate a digraph to a topology by means of the "specialization" relation between points in a topological space: $x$ is a specialization of $y$ if and only if $x$ is in the closure of $\{y\}$. This relation was introduced in a pioneering work by Alexandroff [1] and it has been used by Grothendieck and Dieudonné [5] to characterize the generic points of the irreducible components of a topological space as the maximal points of this binary relation. Reciprocally, we associate a topology $T$ to each digraph $D$ (not necessarily transitive) taking the sets of vertices adjacent to $v$ in $D$, for all vertices $v$, as a subbasis of closed sets for the topology $T$.

In section 2 we use these two associations to make a more profound study of the relations between simple digraphs and their homologous topologies. We also extend the relation between these structures to the functions preserving the structure between these classes of objects.

In section 3 we restrict the study to the particular bijective relation between finite acyclic transitive digraphs and $T_{0}$ topologies. In this context we consider dual concepts to those used in the preceding section to obtain a minimum basis of open sets for the topology $T$ and to prove that the set of the closures of the points in $T$ is the minimum basis of open sets in the dual situation.

This work corresponds to the unpublished first chapter of the author's Ph.D. [6]. 


\subsection{Finite digraphs}

By a digraph we mean a couple $(X, G)$ where $X$ is a finite nonempty set and $G \subset X \times X-\{(x, x): x \in X\}$, (so our digraph has no loops). The elements in $X$ and $G$ are called points and arcs respectively. For an arc $(x, y)$ we will say that $x$ is adjacent to $y$. In the following we will denote by $x y$ an $\operatorname{arc}(x, y)$.

A sequence $x_{1} x_{2} \ldots x_{q} x_{q+1}, q \geq 2$, of distinct points, except $x_{q+1}=x_{1}$, is a cycle if $x_{i} x_{i+1} \in G$ for $i=1,2, \ldots, q$. A digraph is acyclic if it has no cycle. A digraph $(X, G)$ is said to be totally disconnected if $G=\emptyset$.

The non existence of loops in a digraph requires the following correction in the standard concepts of transitivity and antisymmetry.

Definition 1. A digraph $(X, G)$ is said to be transitive if $x y, y z \in G$, with $x \neq z$, then $x z \in G$. A digraph $(X, G)$ is said to be antisymmetric if $x y \in G$, then $y x \notin G$.

We denote the set of digraphs with a set of points $X$ by $\mathcal{G}_{X}$, the subset of transitive digraphs by $\mathcal{G}_{X}^{T}$ and the antisymmetric transitive digraphs by $\mathcal{G}_{X}^{T A}$.

Proposition 2. Let $(X, G)$ be a transitive digraph. Then $(X, G)$ is acyclic if and only if it is antisymmetric.

Proof: An acyclic digraph is antisymmetric because, if there are arcs $x y$ and $y x$, then it must be the cycle $x y x$. Reciprocally, if $x_{1} \ldots x_{q} x_{q+1}$ is a cycle then, by transitivity, $x_{1} x_{q}$ and $x_{q} x_{1}$ are arcs, in contradiction with the antisymmetric property.

\subsection{Finite topological spaces}

Let $X$ be a nonempty set whose elements we will call points. Then, a topology $T$ on $X$ is a set of subsets of $X$, including $\emptyset$ and $X$, that is closed under union and finite intersection and the couple $(X, T)$ is a topological space on $X$. The elements in $T$ are called open sets and their complements closed sets. The largest topology on $X, T=\mathcal{P}(X)$, is called the discrete topology. If $(X, T)$ is a topological space and $A \subset X$, the closure of $A$ is the minimum closed set that contains $A$, and we denote it by $\bar{A}_{T}$, or simply by $\bar{A}$ if there is no possible confusion. We also use some other standard topological concepts such as basis and subbasis of open or closed sets, neighborhood, connection,... as can be see in [8]. 
In the following definition we describe some "separation properties" by means of conditions easily related to each other. In this context, we use $E^{d}$ to denote the derived set of $E \subset X$.

Definition 3. Let $(X, T)$ be a topological space. Then we will say that $(X, T)$ is $T_{0}$ if $\forall x \in X,\{x\}^{d}$ is a union of closed sets or, equivalently, if $\forall x, y \in X$, with $x \neq y$, then $\overline{\{x\}} \neq \overline{\{y\}}$ (Kolmogoroff, 1935). (X,T) is $T_{D}$ if $\forall x \in X,\{x\}^{d}$ is a closed set (Aull-Taron, 1963). $(X, T)$ is $T_{1}$ if $\forall x \in X,\{x\}^{d}=\emptyset$ or, equivalently, if $\forall x \in X, \overline{\{x\}}=\{x\}$ (Frechet, 1906).

It is well known that, in general, $T_{1} \Longrightarrow T_{D} \Longrightarrow T_{0}$, and that the reciprocal is not true.

A topological space $(X, T)$ is finite if the set $X$ is finite. In this case, the following result can be deduced from the above definitions.

Lemma 4. Let $(X, T)$ be a finite topological space. Then

1. $T_{D} \Longleftrightarrow T_{0}$.

2. $T_{1} \Longleftrightarrow T$ is the discrete topology.

Other separation properties, of a general topological space, more restrictive than $T_{1}$ ( $\operatorname{such}$ as $T_{2}, T_{2 a}, T_{3}, T_{3 a}, T_{4}, \ldots$ ) are equivalent, in a finite topological space, to $T_{1}$. In this way, $T_{0}$ is the only relevant separation property in a finite nondiscrete topological space.

\section{Relations between topological spaces and digraphs}

The "specialization" relation between points in a topological space was introduced by Alexandroff [1].

Definition 5. Let $(X, T)$ be a topological space. For each pair of points $x, y \in X$ we will say that $x$ is a specialization of $y$ if and only if $x \in \overline{\{y\}}$.

The specialization relation is a preorder on $X$ : it is obviously reflexive, and is transitive because

$$
x \in \overline{\{y\}} \Longrightarrow \overline{\{x\}} \subset \overline{\{y\}} \text { and } y \in \overline{\{z\}} \Longrightarrow \overline{\{y\}} \subset \overline{\{z\}}
$$

and so $x \in \overline{\{x\}} \subset \overline{\{z\}}$. But, in general, it is not antisymmetric, because in a topology, distinct points can have the same closure, in which case the points are related in both ways. 
This relation permits us to associate a digraph over $X$ to each topological space on the set of points $X$. We denote the set of topological spaces over a same finite set of points $X$ by $\mathcal{T}_{X}$, and the set of $T_{0}$ topological spaces over $X$ by $\mathcal{T}_{X}^{0}$.

Proposition 6. Let $f: \mathcal{T}_{X} \longrightarrow \mathcal{G}_{X}$ be the function given by $f(X, T)=$ $(X, G)$ where $G$ is the set of arcs

$$
G=\{x y: x \in \overline{\{y\}}, x \neq y\}
$$

Then a) $f$ is injective.

b) $f(X, T)=(X, G)$ is a transitive digraph.

c) $f$ is not suprajective.

Proof: a) If $T$ and $T^{\prime}$ are distinct topologies over $X$, then there exists at least a point $x$ with distinct respective closures, that is to say, $\overline{\{x\}}_{T} \neq$ $\overline{\{x\}}_{T^{\prime}}$. Then, there exists a point $y$ with $y \in \overline{\{x\}}_{T}$ and $y \notin \overline{\{x\}}_{T^{\prime}}$ (or vice versa) and so $y x \in G$ and $y x \notin G^{\prime}$ (or vice versa), then $f(X, T) \neq$ $f\left(X, T^{\prime}\right)$.

b) It is a consequence of the transitivity of the specialization relation.

c) $f$ is not suprajective because the nontransitive digraphs do not procced from any topology.

It is also possible to associate a topological space over $X$ to each digraph with set of points $X$ by means of the following procedure.

Definition 7. Let $g: \mathcal{G}_{X} \longrightarrow \mathcal{T}_{X}$ be the function given by $g(X, G)=$ $(X, T)$ where $T$ is the topology over $X$ generated by the subbasis of closed sets

$$
G \downarrow=\{x \downarrow: x \in X\} \text {, where } x \downarrow=\{y: y x \in G\} \cup\{x\}
$$

We also use the notation $g(G)=T$.

Proposition 8. With the notations as above we have

a) $g$ is suprajective.

b) $g \circ f$ is the identity function on $\mathcal{T}_{X}$.

c) $g$ is not injective. 
d) $g$ does not preserve the inclusion relation between digraphs.

e) $f \circ g: \mathcal{G}_{X} \longrightarrow \mathcal{G}_{X}$ is not the identity and nor does it preserve the inclusion.

Proof: a) For any topological space $(X, T) \in \mathcal{T}_{X}$ we consider the digraph $(X, G)=f(X, T)$ and we will prove that $g(X, G)=(X, T)$. We have $G=\{x y: x \in \overline{\{y\}}, x \neq y\}$ and $g(X, G)$ is the topological space $\left(X, T^{\prime}\right)$ whose subbasis of closed sets is $G \downarrow=\{x \downarrow: x \in X\}$ where

$$
\begin{aligned}
x \downarrow & =\{y: y x \in G, x \neq y\} \cup\{x\} \\
& =\{y: y \in \overline{\{x\}}, x \neq y\} \cup\{x\} \\
& =\{x\}
\end{aligned}
$$

so $G \downarrow=\{\overline{\{x\}}: x \in X\}$ and, as this is the subbasis of closed sets of the topology $T$, we have $T=T^{\prime}$.

b) $g \circ f=I_{\mathcal{T}_{X}}$ is a consequence of the construction in a).

Next we give counterexamples proving c), d) and e).

Example 9. For each of the digraphs $(X, G)$ on Figure 1 we construct:

a) the family $G \downarrow$ of unipoint adjacencies $x \downarrow$ (that we will take as a subbasis of closed sets for the topology $T=g(G)$ ),

b) the topology $T$ given by its closed sets,

c) the family $\bar{T}=\{\overline{\{x\}}, x \in X\}$ of unipoint closures of $T$ and

d) the digraphs $f(X, T)$, that we denote by $G^{\prime}$, given in Figure 2. In this way we have $f \circ g(G)=G^{\prime}$ according to the scheme

$$
G \longrightarrow G \downarrow \longrightarrow T \longrightarrow \bar{T} \longrightarrow G^{\prime}
$$
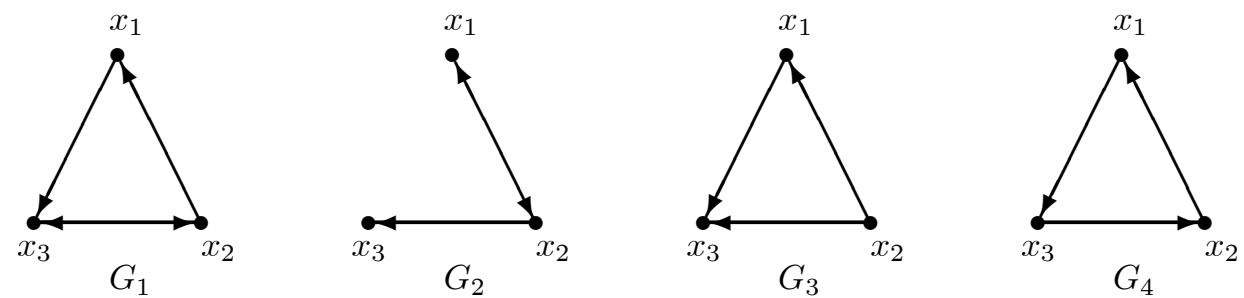

Figure 1

$$
G_{1} \downarrow=\left\{x_{1} \downarrow=\left\{x_{1}, x_{2}\right\}, x_{2} \downarrow=\left\{x_{2}, x_{3}\right\}, x_{3} \downarrow=X\right\}, T_{1}=\left\{\left\{x_{2}\right\},\left\{x_{1}, x_{2}\right\},\right.
$$


$\left.\left\{x_{2}, x_{3}\right\}, X, \emptyset\right\}$ and $\bar{T}_{1}=\left\{\overline{\left\{x_{1}\right\}}=\left\{x_{1}, x_{2}\right\}, \overline{\left\{x_{2}\right\}}=\left\{x_{2}\right\}, \overline{\left\{x_{3}\right\}}=\left\{x_{2}, x_{3}\right\}\right\}$.

$G_{2} \downarrow=\left\{x_{1} \downarrow=\left\{x_{1}, x_{2}\right\}, x_{2} \downarrow=\left\{x_{1}, x_{2}\right\}, x_{3} \downarrow=\left\{x_{2}, x_{3}\right\}\right\}, \quad T_{2}=T_{1}$ and $\bar{T}_{2}=\bar{T}_{1}$.

$G_{3} \downarrow=\left\{x_{1} \downarrow=\left\{x_{1}, x_{2}\right\}, x_{2} \downarrow=\left\{x_{2}\right\}, x_{3} \downarrow=X\right\}, T_{3}=\left\{\left\{x_{2}\right\},\left\{x_{1}, x_{2}\right\}, X, \emptyset\right\}$ and $\bar{T}_{3}=\left\{\overline{\left\{x_{1}\right\}}=\left\{x_{1}, x_{2}\right\}, \overline{\left\{x_{2}\right\}}=\left\{x_{2}\right\}, \overline{\left\{x_{3}\right\}}=X\right\}$.

$G_{4} \downarrow=\left\{x_{1} \downarrow=\left\{x_{1}, x_{2}\right\}, x_{2} \downarrow=\left\{x_{2}, x_{3}\right\}, x_{3} \downarrow=\left\{x_{1}, x_{3}\right\}\right\}, T_{4} \quad$ is the discrete topology and $\bar{T}_{4}=\left\{\overline{\left\{x_{1}\right\}}=\left\{x_{1}\right\}, \overline{\left\{x_{2}\right\}}=\left\{x_{2}\right\}, \overline{\left\{x_{3}\right\}}=\left\{x_{3}\right\}\right.$.
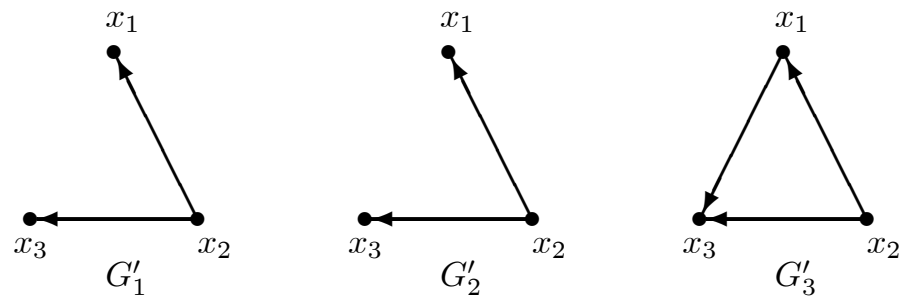

$x_{1}$

Figure 2

Note that $G_{1}$ and $G_{2}$ are non comparable by the inclusion relation but they have the same image by $g$ and by $f \circ g$, proving c) of Proposition 8 .

$G_{1}$ and $G_{3}$ verify $G_{3} \subset G_{1}$ but $f \circ g\left(G_{3}\right) \supset f \circ g\left(G_{1}\right)$, proving e) of Proposition 8.

$G_{1}$ and $G_{4}$ verify $G_{4} \subset G_{1}$ and $T_{4} \supset T_{1}$ and, consequently, $g\left(G_{4}\right) \supset$ $g\left(G_{1}\right)$ and $f \circ g\left(G_{4}\right) \supset f \circ g\left(G_{1}\right)$, proving d) of Proposition 8.

$G_{3}$ and $G_{4}$ are digraphs with "regular behaviour" under $f$ and $g$ as we shall see now.

The non-transitivity is the cause of these anomalies. In the example, only $G_{3}$ is transitive and for this one we have $f \circ g\left(G_{3}\right)=G_{3}$.

Proposition 10. Let $(X, G)$ be the digraph that is a cycle. Then

a) The topology of $g(X, G)$ associated to the cycle $(X, G)$ is the discrete topology.

b) The digraph $f \circ g(X, G)$ associated to the discrete topology $g(X, G)$ is totally disconnected.

Proof: a) Let $X=\left\{x_{1}, \ldots x_{n}\right\}$ and let $x_{1} x_{2} \ldots x_{n} x_{n+1}$ be, with $x_{n+1}=x_{1}$, the cycle. As $x_{i} x_{i+1} \in G$ for $1 \leq i \leq n$, we have $x_{1} \downarrow=\left\{x_{n}, x_{1}\right\}$ and 
$x_{i} \downarrow=\left\{x_{i-1}, x_{i}\right\}$, for $i=2, \ldots, n$. Then $x_{i} \downarrow \cap x_{i+1} \downarrow=\left\{x_{i}\right\}$ is a closed set for $i=1, \ldots, n$ and $g(X, G)$ is thus the discrete topological space on $X$.

b) In the discrete topology we have $\overline{\{x\}}=\{x\}$ for all points $x$, and thus, for $f \circ g(X, G)=\left(X, G^{\prime}\right)$ we have $G^{\prime}=\{x y: x \in \overline{\{y\}}, x \neq y\}=\emptyset$, i.e., the digraph $\left(X, G^{\prime}\right)$ is totally disconnected.

If we restrict the function $g$ to the set of transitive digraphs, the relation between $f$ and $g$ is finally shown to be clear.

Theorem 11. Let $(X, G)$ be a transitive digraph and let $g(X, G)=$ $(X, T)$ be its associated topological space.

Then

$$
x \downarrow=\overline{\{x\}} \quad \text { for each } \quad x \in X
$$

Proof: Because $G \downarrow=\{x \downarrow: x \in X\}$ is a subbasis of closed sets for the topology $T$, the closed sets of $T$ are obtained as intersections of unions of sets $x \downarrow$ and also as unions of intersections of sets $x \downarrow$ (De Morgan's laws). So the minimal closed sets (by inclusion) are obtained as an intersection of sets $x \downarrow$. Then, for each point $y \in X$, we consider the intersection of the closed sets that include $y$ :

$$
C_{y}=\bigcap_{y \in x \downarrow} x \downarrow
$$

As $C_{y}$ is the minimum closed set including $y$, we also have $C_{y}=\overline{\{y\}}$. So, it is sufficient to prove that $C_{y}=y \downarrow$.

$\bigcap_{y \in x \downarrow} x \downarrow \subset y \downarrow$ because $y \downarrow$ is one of the sets in the intersection.

If $z \in y \downarrow$ and $y \in x \downarrow$ (for each $x$ such that $y \in x \downarrow$ ), then, by transitivity, $z \in x \downarrow$ for each $x$ such that $y \in x \downarrow$, then $y \downarrow \subset \bigcap_{y \in x \downarrow} x \downarrow$.

Corollary 12. With the notations as above, the function $f: \mathcal{T}_{X} \longrightarrow \mathcal{G}_{X}^{T}$ is bijective and $f^{-1}=g$.

Proof: It is sufficient to prove that $f \circ g=I_{\mathcal{G}_{X}^{T}}$. The construction process of $f \circ g(X, G)$ from the transitive digraph $(X, G)$ is

$$
G \longrightarrow G \downarrow \longrightarrow T \longrightarrow \bar{T} \longrightarrow f \circ g(X, G)
$$

To prove that $f \circ g(X, G)=G$, it is sufficient to see that $G \downarrow=\bar{T}$ and this was proved in the previous theorem. 
Remark 13. Let $(X, G)$ be a transitive digraph and $(X, T)$ its associated topological space. Then

a) $y \downarrow=\bigcap_{y \in x \downarrow} x \downarrow=\overline{\{y\}}$ that we denote simply by $\bar{y}$.

b) Both the digraph and the topology are known by the family $G \downarrow=$ $\bar{T}=\{\bar{x}: x \in X\}$. In the digraph, $\bar{x}$ is the set $\{y: y x \in G\} \cup\{x\}$. In the topology, $\bar{x}$ is the minimum closed set that contains $x$.

In general, $x \downarrow \subset y \downarrow$ implies $x y \in G$. The reciprocal is not true and characterizes the transitive digraphs.

Proposition 14. Let $(X, G)$ be a digraph. Then $(X, G)$ is transitive if and only if

$$
x y \in G \Longleftrightarrow x \downarrow \subset y \downarrow
$$

Proof: $\Longrightarrow)$ If $(X, G)$ is transitive, $x y \in G$ and if $z \in x \downarrow$, then $z y \in G$ and $z \in y \downarrow$. Obviously $x \in x \downarrow \subset y \downarrow$ implies $x y \in G$.

$\Longleftrightarrow x y, y z \in G$ with $x \neq z \Longleftrightarrow x \downarrow \subset y \downarrow \subset z \downarrow$ with $x \neq z \Longleftrightarrow$ $x z \in G$.

In the digraph $(X, G)$ with $G=\{x y, y x, z x, z y\}$ we have $\bar{x}=\bar{y}=X$ y $\bar{z}=\{z\}$. Thus, in a transitive digraph, it can happen that distinct points, $x \neq y$, have the same closure $\bar{x}=\bar{y}$ and so, in general, $\operatorname{Card}(G \downarrow) \leq$ $\operatorname{Card}(X)$.

Corollary 15. Let $(X, G)$ be a transitive digraph and $x, y$ two distinct points in the digraph. Then

$$
\bar{x}=\bar{y} \Longleftrightarrow x y, y x \in G
$$

The property of a topological space that has distinct closures of all its points characterizes the separation property $T_{0}$ (Definition 3 ). The bijections $f$ and $g$ for transitive digraphs make the properties $T_{0}$ and antisymmetric equivalent. Thus we have the following result:

Theorem 16. Let $(X, G)$ be a transitive digraph and $(X, T)$ its associated topological space. Then, the following conditions are equivalent:

a) $(X, G)$ is antisymmetric.

b) $\bar{x} \neq \bar{y}$, for all $x \neq y$. 
c) $\operatorname{Card}(G \downarrow)=\operatorname{Card}(X)$.

d) $(X, T)$ is $T_{0}$.

e) $(X, G)$ is acyclic (Proposition 2).

Corollary 17. a) There is a bijective correspondence between transitive digraphs and finite topological spaces.

b) There is a bijective correspondence between acyclic transitive digraphs and finite $T_{0}$ topological spaces.

The relation between acyclic transitive digraphs and finite $T_{0}$ topological spaces can be extended to the applications preserving the structure between these classes of objects.

Definition 18. Let $(X, G)$ and $\left(X^{\prime}, G^{\prime}\right)$ be two digraphs. By a digraph morphism $\varphi:(X, G) \longrightarrow\left(X^{\prime}, G^{\prime}\right)$ we understand a function $\varphi: X \longrightarrow$ $X^{\prime}$ such that

$$
x y \in G \Longrightarrow \varphi(x)=\varphi(y) \text { or } \varphi(x) \varphi(y) \in G^{\prime}
$$

The digraph morphism $\varphi$ is an isomorphism if $\varphi$ is bijective and $\varphi^{-1}$ is also a digraph morphism.

Definition 19. Let $(X, T)$ and $\left(X^{\prime}, T^{\prime}\right)$ be two topological spaces. By a continuous function $\varphi:(X, T) \longrightarrow\left(X^{\prime}, T^{\prime}\right)$ we understand a function $\varphi: X \longrightarrow X^{\prime}$ such that

$$
\varphi(\bar{E}) \subset \overline{\varphi(E)} \text { for all } E \subset X
$$

The continuous function $\varphi$ is a homeomorphism if $\varphi$ is bijective and

$$
\varphi(\bar{E})=\overline{\varphi(E)} \text { for all } E \subset X
$$

Lemma 20. Let $(X, T)$ and $\left(X^{\prime}, T^{\prime}\right)$ be two finite topological spaces. Then

a) $\varphi:(X, T) \longrightarrow\left(X^{\prime}, T^{\prime}\right)$ is continuous if and only if $\varphi(\bar{x}) \subset \overline{\varphi(x)}$ for all $x \in X$.

b) $\varphi:(X, T) \longrightarrow\left(X^{\prime}, T^{\prime}\right)$ is a homeomorphism if and only if $\varphi$ is bijective and $\varphi(\bar{x})=\overline{\varphi(x)}$ for all $x \in X$.

Proof: It is a consequence of the properties $\varphi(A \cup B)=\varphi(A) \cup \varphi(B)$ and $\overline{A \cup B}=\bar{A} \cup \bar{B}$ applied to the expression $E=\bigcup_{x \in E} x$. 
Theorem 21. Let $(X, T)$ and $\left(X^{\prime}, T^{\prime}\right)$ be two finite $T_{0}$ topological spaces and $(X, G)$ and $\left(X^{\prime}, G^{\prime}\right)$ their respective associated acyclic transitive digraphs. Let $\varphi: X \longrightarrow X^{\prime}$ be a function between their underlying sets of points. Then

a) $\varphi$ is a continuous function between the topological spaces if and only if $\varphi$ is a digraph morphism between the respective digraphs.

b) $\varphi$ is a homeomorphism between the topological spaces if and only if $\varphi$ is an isomorphism between the respective digraphs.

Proof: If $\varphi$ is a continuous function and $x y \in G$, then

$$
x \in \bar{y} \Longrightarrow \varphi(x) \in \varphi(\bar{y}) \subset \overline{\varphi(y)}
$$

and so either $\varphi(x)=\varphi(y)$ or $\varphi(x) \varphi(y) \in G^{\prime}$.

Reciprocally, if $\varphi$ is a digraph morphism, then, for all $z \in \bar{x}$ with $z \neq x$, we have $z x \in G$ and so, either $\varphi(z)=\varphi(x)$ or $\varphi(z) \varphi(y) \in G^{\prime}$. In both cases we have $\varphi(z) \in \overline{\varphi(x)}$.

Corollary 22. With notations as above and denoting by $\approx$ both the equivalence relations of homeomorphism and isomorphism, we have that the quotient function

$$
f_{/ \approx}: \mathcal{T}_{/ \approx}^{0} \longrightarrow \mathcal{G}_{/ \approx}^{T A}
$$

is bijective.

In [3] it was pointed out that the enumeration of transitive digraphs is a particularly intractable problem. These authors give the number of finite topologies as a function of the number of finite $T_{0}$ topologies by means of the number of partitions of an $n$-set into $m$ parts, also known as Stirling numbers of the second kind. These numbers are currently unknown.

For a history of the enumeration of finite order relations and topologies, and for references about it, see the work of Erné and Stege [4]. These authors describe an algorithm to compute the connected and non connected, $T_{0}$ and non $T_{0}$, topologies on $n$ points for $n \leq 14$. These numbers can also be found in the on-line encyclopedia of integer sequences of Sloane [7].

More recently, Benoumhani [2] has obtained formulas for the number of labeled topologies on $n$ points having $k$ open sets for $k \leq 12$ and for the number of unlabeled $T_{0}$ topologies with $k$ open sets for $n+4 \leq k \leq n+6$. 


\section{Dual digraph}

A finite $T_{0}$ topological space $(X, T)$ and its homologous acyclic transitive digraph $(X, G)$ are both described by the family $\bar{T}=G \downarrow=\{\bar{x}: x \in X\}$ as a subbasis of closed sets (Remark 13).

The topological duality between the concepts of open and closed sets allows us to describe the topology and the digraph over $X$ by the family $\{C \bar{x}: x \in X\}$ as a subbasis of open sets (CA denotes the complementary set of $A \subset X)$. However, these open sets do not form the best subbasis for a simple description of the topological space $(X, T)$, and neither is its homologous digraph concept of interest for a good description of the digraph $(X, G)$.

On the other hand, it is natural to consider a dual concept in the digraph for the $\bar{x}$ (changing the direction of the arrows) and to interpret its meaning in the topology.

Definition 23. Let $(X, G) \in \mathcal{G}_{X}^{T A}$ be an acyclic transitive digraph. For each $x \in X$ we will denote

$$
x \uparrow=\{y, x y \in G\} \cup\{x\} \quad \text { and } \quad x^{*}=\{y, x \in \bar{y}\}
$$

It can be deduced from the definition that $x \in \bar{y} \Longleftrightarrow y \in x^{*}$ and $x^{*}=$ $x \uparrow$.

Lemma 24. With the hypothesis and the notations as above, we have

$$
C \bar{x}=\bigcup_{x \notin y^{*}} y^{*}, \text { for all } x \in X
$$

Proof: We have

$$
z \in C \bar{x} \Longleftrightarrow z \notin \bar{x} \Longleftrightarrow x \notin z^{*}
$$

so $z^{*}$ is among those that unite and, as $z \in z^{*}$, we have $z \in \bigcup_{x \notin y^{*}} y^{*}$.

Reciprocally, if $z \in \bigcup_{x \notin y^{*}} y^{*}$, then there exists $y \in X$ such that $z \in y^{*}$, with $x \notin y^{*}$ and so $x \notin z^{*}$.

Lemma 25. With the hypothesis and the notations as above, we have

$$
x \in C \bar{y} \Longleftrightarrow x^{*} \subset C \bar{y}, \text { for all } x \in X
$$

Proof: If $x \in C \bar{y}$ and $z \in x^{*}$, we have $x y \notin G$ and $x z \in G$. Therefore, by transitivity, we have $z y \notin G$ and $z \in C \bar{y}$. The reciprocal is clear. 
Lemma 26. With the hypothesis and the notations as above, we have

$$
x^{*}=\bigcap_{x^{*} \subset C \bar{y}} C \bar{y}, \text { for all } x \in X
$$

Proof: One inclusion is clear by the nature of the intersections. To prove the other we suppose that there exists $z \in X$ such that $z \in C \bar{y}$ whenever $x^{*} \subset C \bar{y}$ but that $z \notin x^{*}$. Then, we would have $x \in C \bar{z}$ and, by Lemma $25, x^{*} \subset C \bar{z}$. Therefore, we would have $z \in C \bar{z}$, which is absurd.

Note that the formula is preserved when the family of opens $C \bar{y}$ in the intersection is empty, since, if there is no $y$ such that $x^{*} \subset C \bar{y}$, then the digraph is connected and $x$ is the unique minimal point; in consequence, $x^{*}=X$ is the intersection of the empty family.

Remark 27. 1) The formula (3.1) is the translation in terms of digraphs of the topological statement "An open is the intersection of all the opens that contain it". Note that this lemma proves that the sets $x^{*}$ are opens in the homologous topology to the digraph $(X, G)$.

2) Then, Lemma 25 says that $x^{*}$ is the minimum open that contains $x$, because the formula (3.1) admits the versions:

$$
x^{*}=\bigcap_{x \in C \bar{y}} C \bar{y}, \quad x^{*}=\bigcap_{x \notin \bar{y}} C \bar{y}
$$

in which the second members are read as "intersection of the opens that contains $x "$.

Proposition 28. Let $(X, G) \in \mathcal{G}_{X}^{T A}$ be an acyclic transitive digraph and $(X, T) \in \mathcal{T}_{X}^{0}$ its homologous $T_{0}$ topological space. Then the family $T^{*}=$ $\left\{x^{*}: x \in X\right\}$ is a subbasis of open sets for $(X, T)$.

Proof: $\{C \bar{x}, x \in X\}$ is a subbasis of open sets and each $C \bar{x}$ is a union of sets $y^{*}$ (Lemma 24), so $T^{*}$ is also a subbasis.

To consider $T^{*}$ as a subbasis of open sets for $(X, T)$, as opposed to the family $\{C \bar{x}, x \in X\}$, facilitates a digraph interpretation dual to that of $\bar{T}$ as a subbasis of closed sets. We shall now see other topological properties of $T^{*}$ that justify its choice.

Lemma 29. With the notations as above, we have that $T^{*}$ is a basis for $(X, T)$. 
Proof: It is sufficient to prove that any intersection of elements of $T^{*}$ can be written as a union of elements of $T^{*}$. More precisely, let $J$ be any set of indexes, then

$$
\text { if } \bigcap_{J} x_{j}^{*}=\left\{x_{j_{1}}, \ldots, x_{j_{r}}\right\} \text { then } \bigcap_{J} x_{j}^{*}=\bigcup_{k=1}^{r} x_{j_{k}}^{*}
$$

The right hand inclusion is clear, since $x_{j_{k}} \in x_{j_{k}}^{*}$. For the other, let $x \in \bigcup_{k=1}^{r} x_{j_{k}}^{*}$. Then there exists $k$ such that $x \in x_{j_{k}}^{*}$ (or $x_{j_{k}} \in \bar{x}$ ) and, by Proposition 14, we have $\bar{x}_{j_{k}} \subset \bar{x}$. On the other hand, $x_{j_{k}} \in \bigcap_{J} x_{j}^{*}$, then $x_{j} \in \bar{x}_{j_{k}}$, for all $j \in J$. In consequence, $x_{j} \in \bar{x}$, for all $j \in J$, and therefore $x \in \bigcap_{J} x_{j}^{*}$.

Although each $x^{*}$ is the minimum open set that contains $x$, this does not necessarily mean that the intersection of elements of $T^{*}$ is an element of $T^{*}$.

The formula $\bigcap_{J} x_{j}^{*}=\bigcup_{k=1}^{r} x_{j_{k}}^{*}$, even though it has been used to prove Lemma 29, has little practical interest since the union of the right hand side, in general, is not disjoint. Even, some of the open sets that make it up can be redundant. In the next example these observations are made clear.

Theorem 30. With the notations as above, we have that $T^{*}$ is the minimum basis for $(X, T)$.

Proof: We know that $T^{*}$ is a basis and that, for all $x \in X$, we have that $x^{*}$ is the minimum open set including $x$. If $B$ is another arbitrary basis, then the opens of $B$ that contains $x$ are a fundamental system of neighborhoods of $x$ and, so, keeping in mind that $x^{*}$ is a neighborhood of $x$, there exists an open $V \in B$ such that $x \in V \subset x^{*}$. As $x^{*}$ is minimum we have that $V=x^{*}$ and so $x^{*} \in B$, for all $x \in X$.

Corollary 31. Let $B$ be a basis of $(X, T)$. Then $\operatorname{Card}(B) \geq \operatorname{Card}(X)$ and

$$
B=T^{*} \Longleftrightarrow \operatorname{Card}(B)=\operatorname{Card}(X)
$$

This is a consequence of the previous theorem. 
Remark 32. In general, $T^{*}$ is not a minimum subbasis. For the digraph over $X=\{x, y, z\}$ with a set of $\operatorname{arcs} G=\{x z, y z\}$, we have that $T^{*}=$ $\left\{x^{*}=\{x, z\}, y^{*}=\{y, z\}, z^{*}=\{z\}\right\}$ is the minimum basis, but it is not the minimum subbasis, since $\left\{x^{*}, y^{*}\right\}$ is also a subbasis. Nor is $\bar{T}$ the minimum subbasis of closed sets.

Example 33. For the acyclic transitive digraph over $X=\left\{x_{1}, \ldots, x_{7}\right\}$ whose Hasse's diagram (without transitive arcs) is drawed in Figure 3 , the subbasis $\bar{T}$ consists of the closed sets

$$
\begin{gathered}
\bar{x}_{i}=\left\{x_{i}\right\} \text { for } i=1,2,3,4 \text { and } \bar{x}_{5}=\left\{x_{1}, x_{2}, x_{5}\right\}, \\
\bar{x}_{6}=\left\{x_{1}, x_{2}, x_{6}\right\}, \bar{x}_{7}=\left\{x_{1}, x_{2}, x_{3}, x_{5}, x_{7}\right\}
\end{gathered}
$$

The complementary sets of these make up a subbasis of open sets

$$
\begin{gathered}
C \bar{x}_{i}=X-\left\{x_{i}\right\} \text { for } i=1,2,3,4 \text { and } C \bar{x}_{5}=\left\{x_{3}, x_{4}, x_{6}, x_{7}\right\}, \\
\bar{C} x_{6}=\left\{x_{3}, x_{4}, x_{5}, x_{7}\right\}, C \bar{x}_{7}=\left\{x_{4}, x_{6}\right\}
\end{gathered}
$$

and the basis $T^{*}$ consists of the opens

$$
\begin{gathered}
x_{1}^{*}=\left\{x_{1}, x_{5}, x_{6}, x_{7}\right\}, x_{2}^{*}=\left\{x_{2}, x_{5}, x_{6}, x_{7}\right\}, x_{3}^{*}=\left\{x_{3}, x_{7}\right\}, \\
x_{4}^{*}=\left\{x_{4}\right\}, x_{5}^{*}=\left\{x_{5}, x_{7}\right\}, x_{6}^{*}=\left\{x_{6}\right\}, x_{7}^{*}=\left\{x_{7}\right\}
\end{gathered}
$$

These families of sets exemplify the use of the formulas

$x^{*}=\bigcap_{x^{*} \subset C \bar{y}} C \bar{y}, \quad\left(\right.$ Lemma 24) and $C \bar{x}=\bigcup_{x \notin y^{*}} y^{*}$, for all $x \in X$ (Lemma 26)

For the formula $\bigcap_{J} x_{j}^{*}=\bigcup_{k=1}^{r} x_{j_{k}}^{*}$ and the comments in Lemma 29, note that $x_{1}^{*} \cap x_{2}^{*}=\left\{x_{5}, x_{6}, x_{7}\right\} \neq x^{*}$ for all $x \in X$ and

$$
\left\{x_{5}, x_{6}, x_{7}\right\}=x_{5}^{*} \cup x_{6}^{*} \cup x_{7}^{*}=\left\{x_{5}, x_{7}\right\} \cup\left\{x_{6}\right\} \cup\left\{x_{7}\right\}
$$

which proves that not all the opens $x_{j_{k}}^{*}$ are necessary in the union; $x_{j_{k}}^{*}$ being redundant.

Finally, note that $T^{*}$ and $\bar{T}$ are, respectively, a subbasis of closed sets and the minimum basis of open sets for the dual digraph in Figure 4. 


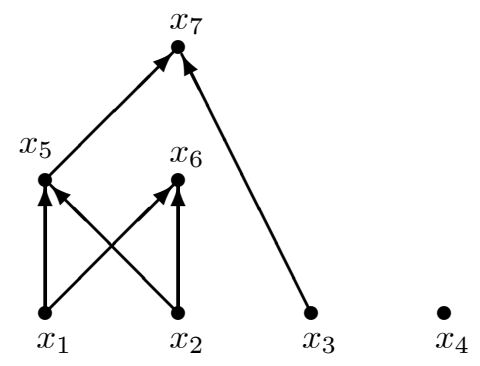

Figure 3. $(X, G)$

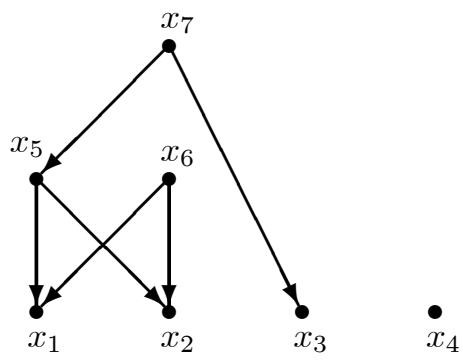

Figure 4. $\left(X, G^{d}\right)$

Definition 34. We call dual digraph of the digraph $(X, G)$, and we denote by $\left(X, G^{d}\right)$ the digraph over $X$ with set of $\operatorname{arcs} G^{d}=\{x y: y x \in G\}$.

It is clear that the transitive and acyclic properties are preserved in the dual digraph. The following conclusions are also clear.

Proposition 35. Let $(X, G)$ be an acyclic transitive digraph and $\left(X, G^{d}\right)$ its dual digraph. Let $(X, T)$ and $\left(X, T^{d}\right)$ be their respective associated $T_{0}$ topological spaces. Then

a) $\bar{T}$ is the minimum basis of open sets for $\left(X, G^{d}\right)$.

b) $T^{*}$ is a subbasis of closed sets (non minimum) for $\left(X, G^{d}\right)$.

c) That is to say, $\bar{T}=T^{d *}$ and $T^{*}=\bar{T}^{d}$.

The continuous functions can also be characterized by the preservation of the open sets $x^{*}$.

Proposition 36. Let $(X, T)$ and $\left(X^{\prime}, T^{\prime}\right)$ be two finite $T_{0}$ topological spaces and let $\varphi: X \longrightarrow X^{\prime}$ be a function. Then $\varphi$ is continuous if and only if $\varphi\left(x^{*}\right) \subset \varphi(x)^{*}$, for all $x \in X$.

Proof: $\varphi$ is continuous if and only if it is a morphism between its homologous digraphs (Theorem 21). This digraph morphism is preserved between the corresponding dual digraphs. Then, keeping in mind that $x^{*}$ is the closure of $x$ in the dual digraph, the result follows easily.

\section{References}

[1] Alexandroff, P. S., Diskrete Rume, Matematiceskii Sbornik (N.S.) 2, pp. 501518, (1937). 
[2] Benoumhani, M., The number of topologies on a finite set, J. of Integer Sequences, Vol. 9, Article 06.2.6, (2006).

[3] Evans, J., Harary, F., Lynn, M., On the computer enumeration of finite topologies, Comm. ACM., 10, pp. 295-298, (1967).

[4] Erné, M., Stege, K., Counting finite posets and topologies topologies, Order, 8, pp. 247-265, (1991).

[5] Grothendieck, A., Dieudonné, J.A., Elémentes de Géometrie Algébrique I, Springer-Verlag, Berlin, (1971).

[6] Marijuán, C., Una teoría birracional para los grafos acíclicos, Ph. D. Dissertation, Universidad de Valladolid, (1988).

[7] Sloane, N. J. A., The on-line encyclopedia of integer sequences published electronically.

[8] Willard, S., General topology, Addison-Wesley, (1970)

\author{
C. Marijuán \\ Dpto. Matemática Aplicada, \\ E.T.S.I. Informática, \\ Paseo de Belén 15, \\ 47011-Valladolid, \\ Spain \\ e-mail : marijuan@mat.uva.es
}

